EPJ Web of Conferences 93,02015 (2015)

DOI: $10.1051 /$ epjconf/20159302015

(C) Owned by the authors, published by EDP Sciences, 2015

\title{
Fast neutron measurements at the nELBE time-of-flight facility
}

\author{
A. R. Junghans ${ }^{a}$, R. Beyer, E. Grosse ${ }^{*}$, R. Hannaske*, T. Kögler*, R. Massarczyk ${ }^{*}$, , R. Schwengner, A. Wagner \\ Helmholtz-Zentrum Dresden-Rossendorf, Postfach 510119, 01314 Dresden, Germany
}

\begin{abstract}
The compact neutron-time-of-flight facility nELBE at the superconducting electron accelerator ELBE of Helmholtz-Zentrum Dresden-Rossendorf has been rebuilt. A new enlarged experimental hall with a flight path of up to $10 \mathrm{~m}$ is available for neutron time-of-flight experiments in the fast energy range from about $50 \mathrm{keV}$ to $10 \mathrm{MeV}$. nELBE is intended to deliver nuclear data of fast neutron nuclear interactions e.g. for the transmutation of nuclear waste and improvement of neutron physical simulations of innovative nuclear systems. The experimental programme consists of transmission measurements of neutron total cross sections, elastic and inelastic scattering cross section measurements, and neutron induced fission cross sections. The inelastic scattering to the first few excited states in ${ }^{56} \mathrm{Fe}$ was investigated by measuring the gamma production cross section with an HPGe detector. The neutron induced fission of ${ }^{242} \mathrm{Pu}$ was studied using fast ionisation chambers with large homogeneous actinide deposits.
\end{abstract}

\section{Introduction}

The compact photo-neutron source nELBE at the Helmholtz-Zentrum Dresden-Rossendorf (HZDR) using a superconducting electron accelerator has been extensively rebuilt. A new time-of-flight experimental hall has been built so that all walls including ceiling and floor are at least $3 \mathrm{~m}$ away from the evacuated neutron beam line. The flight path ranges from about 5 to $10 \mathrm{~m}$. The facility is dedicated to measurements in the fast neutron range. A compact liquid lead circuit is used as neutron producing target. Through this technology the neutron beam intensity is not limited by the heat dissipation inside the target. The technical design including thermomechanical parameters of the liquid lead circuit and the beam dump is discussed in $[1,2]$. The neutron source strength at the nominal beam current of $1 \mathrm{~mA}$ has been calculated to be $10^{13}$ neutrons/s, [2]. The accelerator produces high brilliance beams with variable micropulse repetition rates and duty cycles. The electrons are accelerated up to 30 $\mathrm{MeV}$ in continuous-wave mode by superconducting radio frequency cavities. The maximum average beam current at a micropulse rate of $13 \mathrm{MHz}$ is $1 \mathrm{~mA}$. For typical timeof-flight measurements the repetition rate is reduced to $100-400 \mathrm{kHz}$. The excellent time structure of the electron beam with a micro-bunch length of about 5 ps together with compact size of the neutron producing target allows us to use short flight paths with a high resolution detection system. Figure 1 shows the floor plan of the new neutron time-of-flight facility in the Center for High Power Radiation Sources of HZDR.

The electron beam passes through a beryllium window mounted on a stainless-steel vacuum chamber and hits the radiator, consisting of a molybdenum channel confining the liquid lead. The channel has a rhombic cross section with $11 \mathrm{~mm}$ side length. The electrons generate bremsstrahlung photons which release neutrons in secondary $(\gamma, n)$ reactions on lead. These leave the radiator almost isotropically, while the angular distributions of electrons and photons are strongly forward-peaked. The collimator and the resulting neutron beam properties at the experimental area have been optimized using MCNP in order to maintain the correlation of time-of-flight and neutron energy. The collimator of $2.6 \mathrm{~m}$ length contains three replaceable elements of lead and borated polyethylene that are mounted inside a precision steel tube [2]. The new neutron producing target and collimator have the same design and dimensions as before the extension of the facility, consequently a very similar neutron spectrum and spatial beam profile is expected [3].

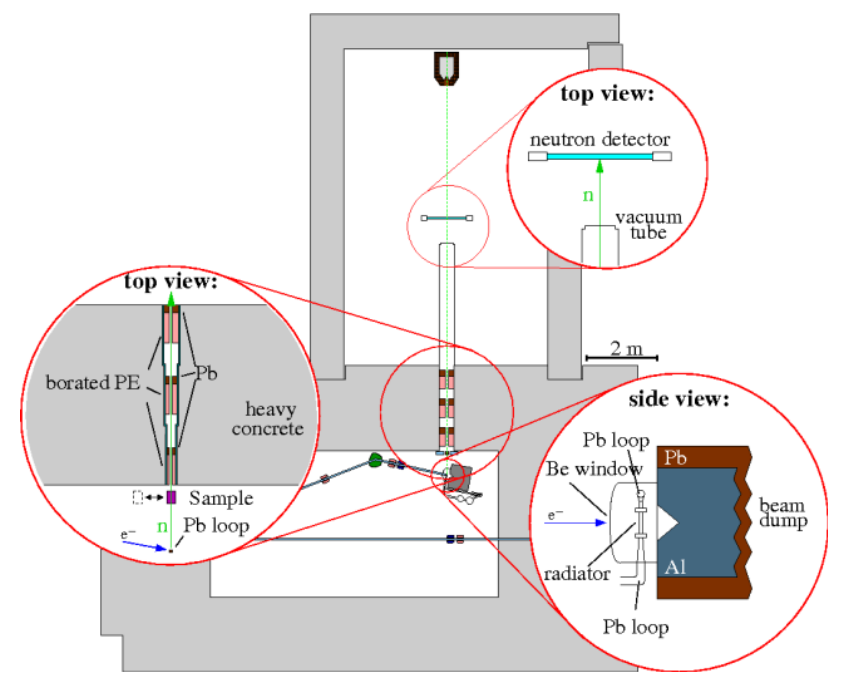

Figure 1: Floor plan of the new nELBE neutron source and low background experimental hall. The insets show the details of neutron producing target, collimator and of a plastic scintillator used for transmission measurements.

The neutron time of flight range is typically from $100 \mathrm{~ns}$ to about $2.5 \mu \mathrm{s}$ for an energy range of $100 \mathrm{keV}$ to $7 \mathrm{MeV}$ and a spectral rate of $6^{*} 10^{3} \mathrm{n} /\left(\mathrm{cm}^{2} \mathrm{~s} \mathrm{MeV}\right)$. The neutron spectral rate as shown in Figure 2, has been measured [3] with the ${ }^{235} \mathrm{U}$ fission chamber $\mathrm{H} 19$ on loan from Physikalisch-Technische Bundesanstalt, Braunschweig (PTB). The time resolution of the fission chamber was estimated from the width of the photofission peak due to bremsstrahlung to be $3.8 \mathrm{~ns}$ (FWHM). Some absorption

\footnotetext{
a Corresponding author: A.Junghans@hzdr.de; ${ }^{b}$ present address: Los Alamos Nat. Lab., U.S.A. * also at Technische Universität Dresden, Institut für Kern- und Teilchenphysik, Dresden, Germany
} 
dips at $78,117,355,528,722,820 \mathrm{keV}$ due to scattering from resonances in ${ }^{208} \mathrm{~Pb}$ can be seen. Emission peaks at $40,89,179,254,314,605 \mathrm{keV}$ are due to near threshold photoneutron emission from ${ }^{208} \mathrm{~Pb}$.

Experimental setups have been built to study the neutron total cross section by transmission measurements, elastic and inelastic scattering, and neutron-induced fission.

\section{Neutron total cross sections}

The neutron total cross section of ${ }^{197} \mathrm{Au}$ in the fast range starting at $100 \mathrm{keV}$ has been measured at nELBE and at GELINA in the range up to $100 \mathrm{keV}$. A precise measurement of the neutron total cross section is relevant for the evaluation of the neutron capture cross section of $\mathrm{Au}$, which is an important standard. The effective neutron total cross section can be determined from the measured neutron transmission $T$ by the well known relation

$$
T=\frac{R_{\text {in }}}{R_{\text {out }}}=\exp \left(-n l \sigma_{\text {tot }}\right)
$$

where $R_{\text {in }}, R_{\text {out }}$ are the background and dead-time corrected count rates in the detector with the sample in and out of the beam, respectively. The areal atom density is given by $n l$ and $\sigma_{t o t}$ is the effective neutron total cross section that can be derived from the measured transmission data. For this measurement samples of ${ }^{197} \mathrm{Au}$ $(n l=0.09422 \pm 0.00014)$ and ${ }^{\text {nat }} \mathrm{Fe}(n l=0.16959 \pm$ 0.00027 ) have been mounted in the target ladder at the entrance of the collimator. To compensate for possible long term drifts of the electron beam intensity the targets have been cycled in and out of the beam in intervalls of $10 \mathrm{~min}$ (empty)/20 min (target in). The total measurement time was 107 hours (including measurement of a tungsten sample).

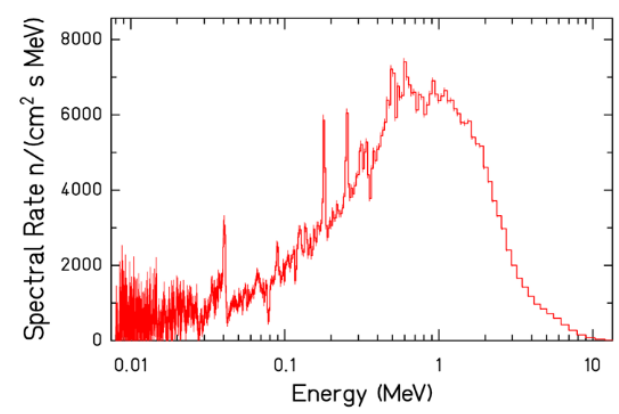

Figure 2 Spectral rate of neutrons measured with an electron beam current $\mathrm{I}_{\mathrm{e}-}=15 \mu \mathrm{A}(200 \mathrm{kHz}$ repetition rate $)$, energy $\mathrm{E}_{\mathrm{e}-}=$ $31 \mathrm{MeV}$ and flight path $1=618 \mathrm{~cm}$.

A $20 \mathrm{~cm}$ long, $1 \mathrm{~cm}$ thick, $4.2 \mathrm{~cm}$ wide plastic scintillator bar coupled directly on both ends to R2059 phototubes (PMT) from Hamamatsu has been used to detect the neutrons. As the instantaneous count rate is highest during the bremsstrahlung peak, the deadtime correction is time-of-flight dependent $[3,5]$. By using the new TDC acquistion mode as described below the deadtime correction for the empty target setting (highest count rate) is now only $4.5 \%$. Both PMTs are triggered with a very low threshold in the single-photo-electron peak. The data acquisition is triggered by a coincidence of both PMTs, thus the generally uncorrelated noise is suppressed, but even low energy recoil protons can be detected. The threshold of this detector is below $10 \mathrm{keV}$ neutron energy [4]. The electron beam intensity was reduced to the sub$\mu A$ range with a micropulse repetition rate of $101 \mathrm{kHz}$ to reach a detector count rate of $4.7 \mathrm{kHz}$ (empty sample beam). This corresponds to a neutron count rate of approximately $170 \mathrm{~s}^{-1}$. Thus, only about every twentieth accelerator pulse is measured in the scintillator. To determine the neutron transmission and the total cross section from the measured time-of-flight distribution several corrections to the measured count rates have to be considered. Most important is the subtraction of time-offlight uncorrelated background. In this experiment this is suppressed by a $1 \mathrm{~cm} \mathrm{~Pb}$ shield around the detector. Nearly $90 \%$ of the background count rate are not induced by the beam. Figure 3 shows a time-of-flight spectrum with the $\mathrm{Au}$ absorber in the beam. The spectrum was accumulated with a short gate on the time-difference spectrum of both PMTs to select events that occur in the center region of the detector inside the neutron beam spot and suppress false coincidences. The small peak before the bremsstrahlung peak might be due to false coincidences in the plastic scintillator.

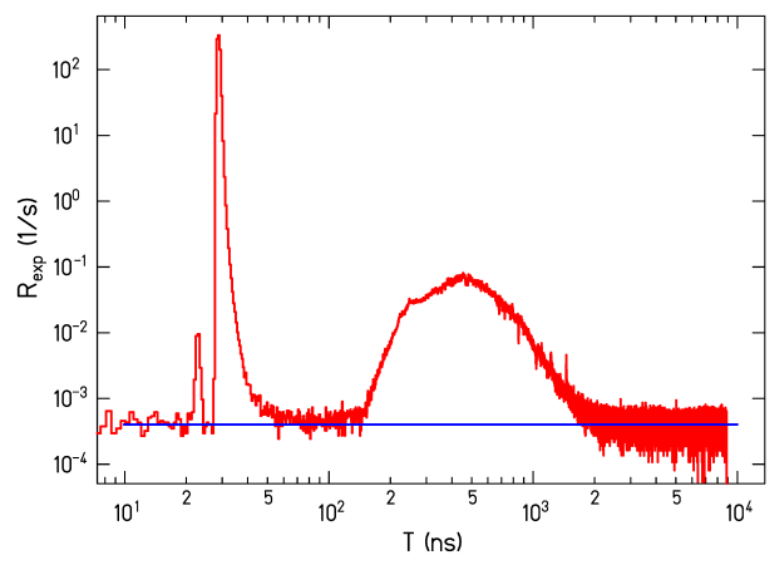

Figure 3: Neutron time-of-flight spectrum for the transmission of a nat $\mathrm{Au}$-sample. The bremsstrahlung peak at about $29 \mathrm{~ns}$ time-of-flight has a FWHM of $1.0 \mathrm{~ns}$ and an integral count rate of $440 \mathrm{~s}^{-1}$. The neutron distribution has an integral count rate of about $30 \mathrm{~s}^{-1}$. The constant, uncorrelated background (blue line) is about $4 \mathrm{~s}^{-1}$.

The dead time of the data acquisition system has been reduced significantly compared to a former measurement at the old nELBE setup [5]. A multi-hit multi-event TDC CAEN V1290N is used to measure the time of the plastic scintillator signals relative to the accelerator radio frequency. It can continue to measure during the read out cycle of the MBS data acquisition system (GSI, Darmstadt). A $3 \mu$ s deadtime is induced for each PMT signal to suppress triggering of afterpulses. The veto length is measured event-wise with a CAEN V1495 general purpose VME board using its $40 \mathrm{MHz}$ internal clock. 


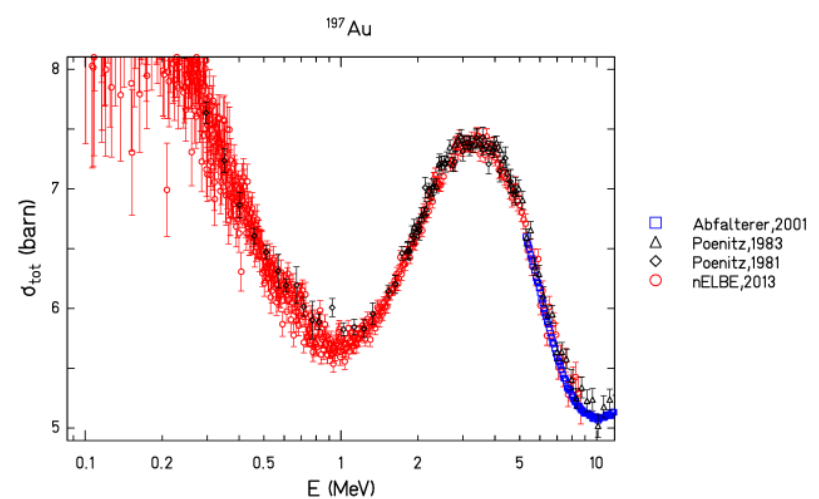

Figure 4: Neutron total cross section of ${ }^{197} \mathrm{Au}$ measured at nELBE compared with measurements from Abfalterer et al.[6] and Poenitz et al. [7,8].

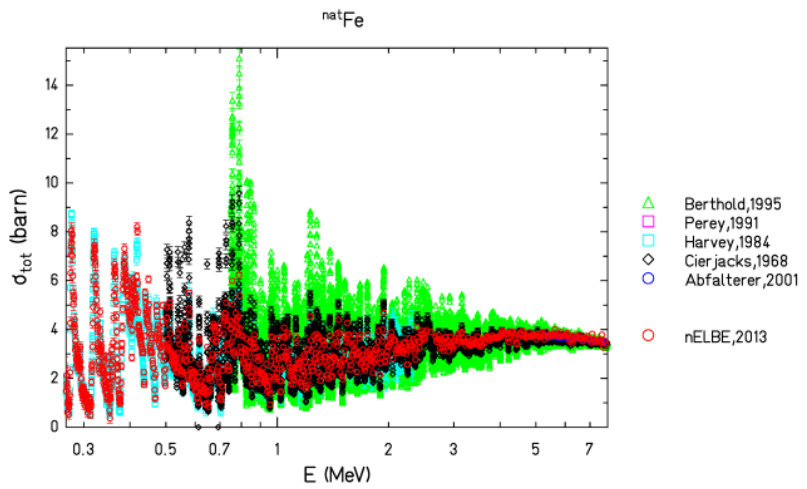

Figure 5 :Neutron total cross section of ${ }^{\text {nat }} \mathrm{Fe}$ measured at nELBE (red circles) with a time-of-flight binsize of $0.977 \mathrm{~ns}$. The data are taken from the EXFOR data base, see refs.[6,9,10,11,12].

The neutron total cross section measured for ${ }^{197} \mathrm{Au}$ is shown in Figure 4. The data are in very good agreement with the measurements done at the WNR facility at Los Alamos Nat. Lab. [6] and at the Argonne Fast-Neutron Generator $[7,8]$. The total cross sections measured previously at the old nELBE setup were $2-3 \%$ higher. They were measured with a data acquisition that had a live time of only $56 \%$ in the empty target case [5]. As this is a rather large correction, a small error in it, could explain this difference. In Figure 5 the neutron total cross section measured for ${ }^{\text {nat }} \mathrm{Fe}$ is shown in comparison with results from other experiments. At low neutron energy around $0.3-0.5 \mathrm{MeV}$ the resolution in the nELBE measurement with a flight path of $8.521 \mathrm{~m}$ is very similar to the resolution from a measurement of Harvey et al., at ORELA using a flight path of $80.263 \mathrm{~m}$ [9]. The absolute agreement with Abfalterer et al. [6] and with the highresolution data from IRMM [12] is very good.

\section{Photon production cross section measurement of ${ }^{56} \mathrm{Fe}$}

${ }^{n a t} \mathrm{Fe}$ is an important structural and fuel cladding material for innovative reactor systems and inelastic scattering has a big influence on the neutron energy spectrum. The reaction ${ }^{56} \mathrm{Fe}\left(\mathrm{n}, \mathrm{n}^{\prime}\right)$ is included in the High Priority Request list of the OECD Nuclear Energy Agency. The inelastic scattering reaction ${ }^{56} \mathrm{Fe}\left(\mathrm{n}, \mathrm{n}^{\prime}\right)$ was studied at nELBE by measuring the $\gamma$-rays emitted as a function of time-of-flight. The target was a cylinder of natural iron with a diameter of $20 \mathrm{~mm}$ and thickness of $8 \mathrm{~mm}$ with a flight path of $6.2 \mathrm{~m}$. An HPGe detector $(60 \%$ relative efficiency) located under 125 degrees to the neutron beam and a distance of $20 \mathrm{~cm}$ from the target was used to measure the $\gamma$-ray energy spectrum.

The time-of-flight was determined from the HPGe detector and the accelerator RF signals. A time resolution of $10 \mathrm{~ns}$ (FWHM) was determined from the width of the bremsstrahlung peak. The background was subtracted with the help of $\gamma$-spectra that were taken with the target out of the beam.

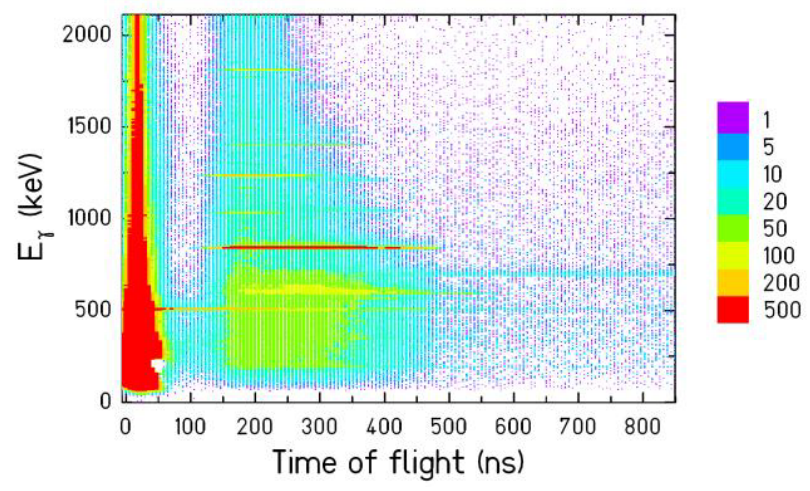

Figure $6 \gamma$-ray energy vs. time-of-flight spectrum measured with an HPGe detector (60\% relative efficiency). The absolute time-of-flight can be obtained from the bremsstrahlung peak and the flight path length.

This also helped to suppress a possible time-of-flight uncorrelated background of $\gamma$-rays from the $\beta$-decay to excited states of ${ }^{56} \mathrm{Fe}$ from the activation reaction ${ }^{56} \mathrm{Fe}(\mathrm{n}, \mathrm{p}){ }^{56} \mathrm{Mn}$. Figure 6 shows the $\gamma$-ray energy vs. timeof-flight histogram. $\gamma$-rays from excited states in ${ }^{56} \mathrm{Fe}$ are clearly visible, e.g. $847 \mathrm{keV}, 1238 \mathrm{keV}$, and $1811 \mathrm{keV}$. To determine the inelastic scattering yield for the first few excited states the feeding from higher-lying transitions must be subtracted. This can be done, as the gamma-decay scheme is well known. The cross section was determined using the neutron spectral rate as shown in Figure 2 including corrections for the scattering of neutrons in air and the materials in between the fission chamber and the target position, the attenuation of $\gamma$-rays from inelastic scattering and multiple inelastic scattering of neutrons in the Fe sample, for details see Refs. [3,11].

From the photon production cross section under 125 degrees the angle integrated scattering cross section can be determined. The contribution of higher order Legendre polynomials is small and not included in the data shown here [13]. The inelastic scattering cross section for ${ }^{56} \mathrm{Fe}\left(\mathrm{n}, \mathrm{n}^{\prime}\right)$ to the first $2^{+}, 4^{+}$and $6^{+}$states is shown in Figure 7 . Due to the limited time-of-flight resolution of the HPGe detector the rather sharp resonant structure of the inelastic scattering cross section e.g. to the first $2^{+}$ state cannot be resolved in this experiment. The data 
shown here are in good agreement with a recent highresolution time-of- flight measurement using the GAINS setup at IRMM with a flight path of $200 \mathrm{~m}$ [14].

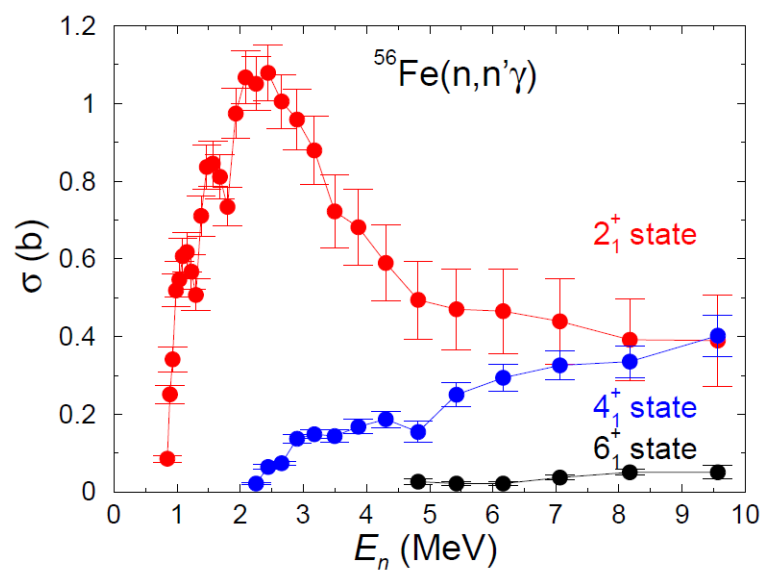

Figure 7 Inelastic scattering cross section measured for the first $2^{+}, 4^{+}$and $6^{+}$states in ${ }^{56} \mathrm{Fe}[13]$.

\section{Neutron-induced fission cross section measurement of ${ }^{242} \mathrm{Pu}$}

Plutonium isotopes are formed through neutron capture in the fuel of nuclear power reactors. The even neutronnumber isotopes, e.g. ${ }^{242} \mathrm{Pu}$ cannot efficiently fission in a thermal neutron spectrum, thus they form a part of the long-lived nuclear waste. In a fast neutron spectrum in principle all actinides incl. ${ }^{242} \mathrm{Pu}$ can fission. Sensitivity studies with reactor simulation codes have shown that the experimental uncertainty of e.g. the ${ }^{242} \mathrm{Pu}$ fission cross section limits the accuracy of integral reactor parameters [15]. Therefore, improved measurements are of interest.

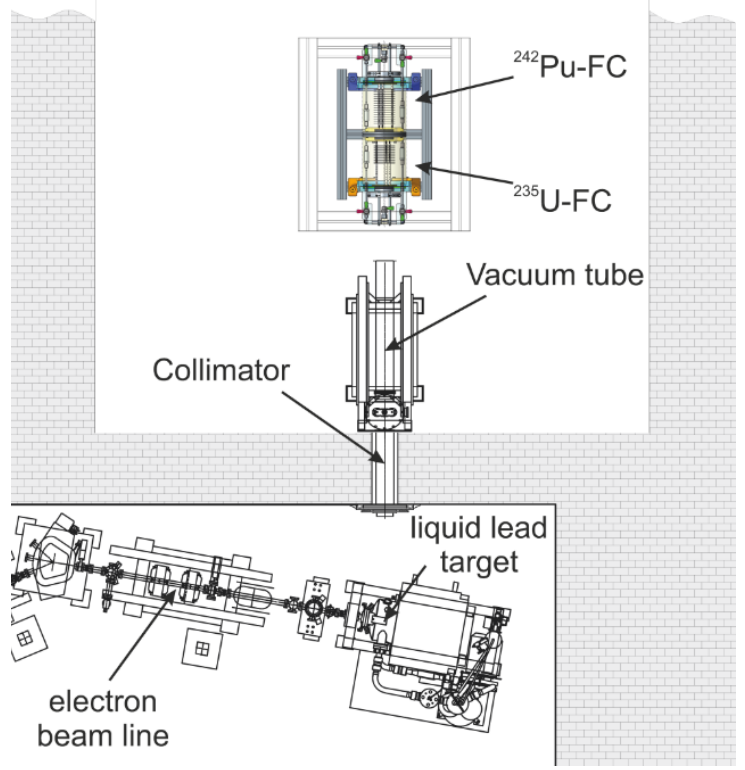

Figure 8 Set up of the fission experiment at nELBE (not to scale). At the exit of the evacuated beam tube is the setup with two fission chambers. First a ${ }^{235} \mathrm{U}$ fission chamber for determination of the neutron spectral rate and behind the fission chamber with 8 large area ${ }^{242} \mathrm{Pu}$ deposits.
The fission cross section measurement of ${ }^{242} \mathrm{Pu}$ is complicated by the fact that the $\alpha$-radioactivity of the fission sample is significant and can induce a background that limits the detection of fission reactions. Two parallelplate fission chambers with ${ }^{235} \mathrm{U}$ and ${ }^{242} \mathrm{Pu}$ deposits have been developed at HZDR. The actinide deposits were produced at the Institute for Nuclear Chemistry of the University of Mainz by molecular plating [16]. The homogeneity of these very thin actinide targets $\left({ }^{235} \mathrm{U}: \mathrm{n}_{\mathrm{A}}\right.$ $\approx 450 \mu \mathrm{g} / \mathrm{cm}^{2},{ }^{242} \mathrm{Pu}: \mathrm{n}_{\mathrm{A}} \approx 125 \mu \mathrm{g} / \mathrm{cm}^{2}$ ) was improved by using titanium coated silicon wafer (thickness $400 \mu \mathrm{m}$ ) as a homogeneous backing material [16]. To avoid uncertainties related to beam inhomogeneity, a sample diameter of $74 \mathrm{~mm}$ was chosen that is larger than the nELBE beam diameter. Both fission chambers were mounted face-to-face within the neutron beam, whereby the uranium chamber was placed closer to the neutron source. The flight path of the neutrons was between 596.5 $\mathrm{cm}$ for the first uranium layer and $639.9 \mathrm{~cm}$ for the last plutonium layer. A sketch of the experimental setup is shown in Figure 8. Both fission chambers were operated in the forward biasing mode, which means that the anode (a blank electrode made from tantalum, thickness 100 $\mu \mathrm{m})$ is read out, whereas the actinide deposits compose the cathode. The anode is connected to a charge sensitive preamplifier, built at HZDR and read out by a QDC and TDC combination to record charge and timing information simultaneously. To reduced sensitivity to pile-up of $\alpha$-particle induced signals in the ionisation chamber, especially in the case of ${ }^{242} \mathrm{Pu}$, the 8 anodes were read out by individual data acquisition channels. Compared to conventional charge sensitive preamplifiers, this device enables total signal lengths in the order of 200- 400 ns. Figure 9 shows the charge spectrum of a ${ }^{242} \mathrm{Pu}$ deposit measured with a QDC. The separation of $\alpha-$ induced signals and fission-induced signals is very good. This is in part due to the anode-cathode distance of $9 \mathrm{~mm}$ that allows a higher energy deposition of the fission fragments in the P10 counting gas.

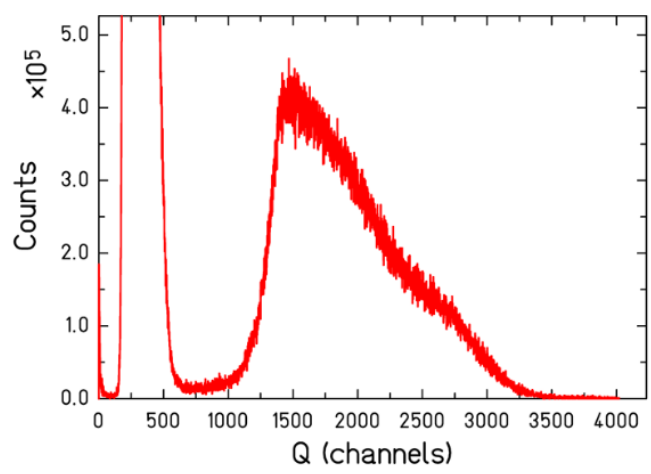

Figure 9 Charge spectrum of a ${ }^{242} \mathrm{Pu}$-fission chamber deposit. The signals from $\alpha$-radioactivity (ch. 600 and below) are well separated from signals due to fission fragments.

By putting gates on the pulse-height range of the fission fragments in the charge spectrum e.g. as shown in Figure 9, the time-of flight spectra of the neutron-induced and 
photo-fission can be measured, as shown in Figure $\mathbf{1 0 .}$ The time resolution of the ${ }^{242} \mathrm{Pu}$ fission chamber is typically $2.5 \mathrm{~ns}$ (FWHM) and was deduced from the width of the photofission peak. As ${ }^{242} \mathrm{Pu}$ is mostly fissile by fast neutrons the time-of-flight spectrum ends at a time of flight corresponding to about $600 \mathrm{keV}$ neutron kinetic energy, whereas the ${ }^{235} \mathrm{U}$ time-of-flight spectrum extends down toward the end of the neutron spectrum produced at nELBE. The neutron-induced fission cross section can be determined relative to the one of ${ }^{235} \mathrm{U}$ that is a standard in the fast neutron range above $0.150 \mathrm{MeV}$.

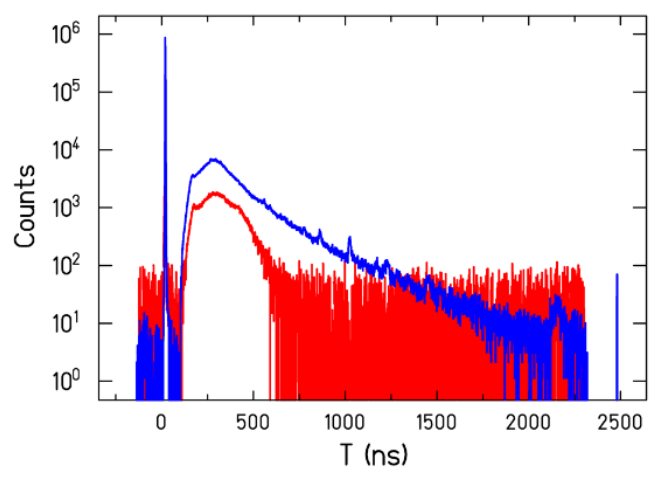

Figure 10 Time-of-flight spectra of one channel of the ${ }^{242} \mathrm{Pu}$ (red) and the ${ }^{235} \mathrm{U}$ (blue) fission chamber. An uncorrelated, constant background from spontaneous fission of ${ }^{242} \mathrm{Pu}$ was subtracted. The spectra where accumulated under a pulse-height condition that a fission fragment was registered in the fission chamber.



Figure 11 Relative neutron-induced fission cross section of ${ }^{242} \mathrm{Pu}$ determined at nELBE (blue data points). The data are shown together with two selected datasets from Tovesson et al.[17] (red) and Weigmann et al. (green)[18]. The evaluated nuclear data from the ENDF/B-VII.1 database is shown with a solid black line together with its gray shaded uncertainty band.

Using the well-known spontaneous fission rate of ${ }^{242} \mathrm{Pu}$ [19] the product of number of target atoms and the intrinsic detection efficiency for a fission fragment in the fission chamber cancels out in the relative fission cross section. This makes the correction for neutron scattering in the fission setup the most importane one. The correction factor is best determined by a neutron transport simulation in realistic geometry. The correction factor to be applied to the measured cross section can be determined by the ratio of the simulated fission rate for a completely massless setup (product of the neutron spectral rate and the fission cross section) and the simulated fission rate taking into account all material that scatters neutrons into the actinide deposits (e.g. backings, windows, fission chamber housing, air, etc.) The simulation using MNCP and GEANT4 are currently ongoing. The preliminary data shown in Figure 11 still have to be corrected for this effect.

The neutron transmission measurements are a collaboration with JRC-IRMM, Geel, Belgium and KAERI, Korea. We would like to thank Manfred Sobiella and Klaus Heidel for the support in the construction of the fission chamber and the preamplifiers. We also thank Andreas Hartmann for the continuous support in the preparation of our experiments. Special thanks go to Alessio Vascon and Klaus Eberhardt from the Johannes Gutenberg University of Mainz for their efforts in the production of the target samples. Thanks also go to the ELBE accelerator crew and all of the colleagues, who have participated during the experiments. This work was supported by the European Commission within the Seventh Framework Programme through Fission-2010ERINDA (project no.269499) and by the German Federal Ministry for Education and Science (TRAKULA project, contract number 02NUK13A).

\section{References}

1. A. Altstadt et al., Ann. Nucl. Energy 34, 36 (2007)

2. J. Klug et al., Nucl. Inst. Meth. A 577, 641 (2007)

3. R. Beyer et al., Nucl. Inst. Meth. A 723, 151 (2013)

4. R. Beyer et al., Nucl. Inst. Meth. A 575, 449 (2007)

5. R. Hannaske et al., Eur. Phys. J. A 49, 137 (2013)

6. W. P. Abfalterer et al., Phys. Rev. C 63, 044608 (2001). (EXFOR:subentries 13753019, 13753026)

7. W. Poenitz, et al., Nucl. Sci. Eng. 78, 333 (1981) (EXFOR:subentry 10935003)

8. W. Poenitz and J. Whalen, Report ANL/NDM-80 (1983). (EXFOR subentry: 12853051)

9. F. G. Perey, J. A. Harvey, and N. W. H i 11 Report to the DOE Nuclear Data Committee, 1984, INDC(USA)-93/L, 142 (EXFOR: 13727003)

10. S. Cierjacks, report KFK-1000 (1968) (EXFOR : 20010007)

11. C.M. Perey et al., Proc. Int. Conf. on Nucl.Data for Sci. and Technol., Jülich 1991, p.41 (1991) (EXFOR subentry : 13511002)

12. K. Berthold (EXFOR subentry : 22276002

13. R. Beyer, et al., Nucl. Phys. A 927, 41 (2014).

14. A. Negret et al., Phys. Rev. C 90, 034602 (2014)

15. M. Salvatores, NEA/WPEC 26 report, Uncertainty and target accuracy assessment for innovative systems using recent covariance data evaluations, OECD/NEA (2008).

16. A. Vascon et al., Appl. Rad. and Iso. 95, 36 (2015)

17. F. Tovesson et al., Phys. Rev. C 79, 014613 (2009) (EXFOR: 14223003)

18. H. Weigmann (1984) EXFOR: 21931002

19. P. Salvador-Castineira, et al., Phys. Rev. C 88, 064611 (2013) 
